\title{
DEBATES
}

\section{La Consolidación de un Nuevo Sistema de Partidos en Uruguay}

\author{
The Consolidation of a New Party System in Uruguay
}

\section{Daniel Buquet \\ Rafael Piñeiro}

\section{Resumen}

A pesar de su alto grado de institucionalización y de la relativa estabilidad de la competencia electoral, el sistema de partidos uruguayo ha sufrido una significativa transformación en los últimos cuarenta años. Este artículo analiza el proceso de cambio del viejo equilibrio bipartidista, que se mantuvo hasta la década de 1960, hasta el nuevo equilibrio tripartidista que se expresó en las elecciones de 2009. Para ello, se estudia la evolución de los resultados electorales, de la volatilidad y de la fragmentación, además del cambio de reglas que permitió la consolidación del nuevo sistema de partidos. Este trabajo permite comprender, desde una perspectiva institucionalista, la forma que adoptan las transformaciones de los sistemas de partidos en países altamente institucionalizados como Uruguay. La hipótesis es que los cambios producidos en el sistema de partidos, originados en shocks exógenos, se procesan gradualmente y se estabilizan a través del cambio de las reglas electorales.

\section{Palabras clave}

Sistemas de Partidos; Institucionalización; Sistema Electoral; Equilíbrio; Uruguay.

\begin{abstract}
Despite its high degree of institutionalization and the relative stability of electoral competition, the Uruguayan party system has undergone a significant transformation in the last forty years. This article analyzes that change, from the bipartisan equilibrium that remained until the 1960's, to the new three party system equilibrium expressed in the 2009 elections results. To achieve that goal it examines the evolution of electoral results, electoral volatility, and party fragmentation, as well as the change of rules that allowed the consolidation of the new party system. This perspective helps to understand, from an institutionalist view point, the way in which highly institutionalized party systems change. The central hypothesis states that changes in party systems, originated by exogenous shocks, are processed gradually through changes in electoral rules that help to stabilize the system in a new equilibrium.
\end{abstract}

\section{Keywords}

Party Systems; Institutionalization; Electoral Systems; Equilibrium; Uruguay. 


\section{Introducción 1}

Uruguay cuenta con uno de los sistemas de partidos más institucionalizados de América Latina. Esta afirmación es aceptada de forma prácticamente unánime en la literatura especializada y el objetivo de este artículo no es ponerla en cuestión. Por el contrario, en este trabajo se busca aprovechar la oportunidad que brinda el caso uruguayo, para comprender de forma precisa en qué consiste y cómo se produce la institucionalización de un sistema de partidos. En ese sentido se pretende contribuir a llenar un laguna en el conocimiento sobre la materia, ya que en lo sustancial, la disciplina no ha avanzado mucho más allá de una caracterización que permite distinguir a los sistema institucionalizados de los que no lo están.

Uruguay renovó la totalidad de sus cargos de gobierno electivos entre junio de 2009 y mayo de 2010, utilizando por tercera vez el sistema electoral reformado en 1997. El peculiar ciclo electoral uruguayo incluye, cada cinco años desde 1999, entre tres y cuatro convocatorias a la ciudadanía a lo largo de un periodo de menos de un año: i) en primer lugar, en el mes de junio, se realizan las elecciones internas de los partidos y las primarias presidenciales; ii) luego, en octubre, se lleva a cabo la elección general, que comprende las dos cámaras del Poder Legislativo y la primera vuelta presidencial; iii) a continuación, en caso de que ningún candidato haya obtenido la mayoría absoluta en la primera vuelta, se realiza la segunda vuelta presidencial en noviembre; y iv) finalmente, en mayo del año siguiente, se desarrolla la elección de autoridades subnacionales.

Aunque en esta última ocasión, los resultados electorales no ofrecieron cambios muy significativos con respecto a los ocurridos cinco años atrás, una mirada atenta y centrada en el largo plazo permite apreciar que estas elecciones muestran una transformación sustancial de la pauta de evolución del sistema de partidos uruguayo en los últimos casi cuarenta años. Las elecciones anteriores, en 2004 y 2005, fueron evaluadas como las del mayor cambio político, ya que por primera vez en la historia del país un partido que no era ni el Partido Colorado (PC) ni el Partido Nacional (PN) llegaba al gobierno. Pero el cambio político que cristalizó en 2004 es la culminación de un proceso que comenzó en 1971 cuando se formó el Frente Amplio (FA). A lo largo de ese proceso el FA fue creciendo sistemáticamente a costa del caudal electoral de los partidos tradicionales. Elección tras elección se observaba un significativo cambio en las preferencias de la ciudadanía que crecientemente favorecía a la izquierda política. Pero en 2009 no se pudo observar prácticamente ninguna modificación relevante en el comportamiento electoral uruguayo. En consecuencia, al

\footnotetext{
${ }^{1}$ Este artículo está elaborado sobre la base de Buquet y Pińeiro (2010, 2011b y 2013).
} 
observar el mediano plazo la elección de 2009 es la primera de una nueva era. La elección que abre una nueva normalidad, diferente a la del bipartidismo tradicional que se quebró en 1971. Una normalidad, un nuevo equilibrio del sistema, reforzada por las reglas electorales que cambiaron en 1997.

La nueva realidad política de Uruguay, como en los sistemas de partidos institucionalizados, es procesada por los viejos actores. Las continuidades en cuanto a las referencias políticas, a los liderazgos y a las posiciones de los partidos respecto a los temas políticos centrales, se mezclan con los cambios en las dinámicas de competencia política y la forma en que los electores reaccionan ante el desempeño de los gobiernos y los partidos.

En este artículo se describe la evolución del sistema de partidos uruguayo y se propone una explicación para la estabilidad de su configuración actual. En primer lugar se presentan las características del sistema de partidos uruguayo en el mediano plazo. Luego, se estudian los cambios de tendencias que dan lugar a ver a esta elección como la primera de una nueva era y se delinean las características del nuevo equilibro del sistema de partidos uruguayo sobre la base de las reglas electorales vigentes.

\section{La evolución del sistema político uruguayo en el mediano plazo}

El sistema de partidos uruguayo es uno de los que cuentan con mayores niveles de institucionalización en América Latina y también ha mostrado una evolución muy predecible a lo largo de las últimas décadas. La literatura comparativa, desde la formulación misma del concepto de institucionalización (MAINWARING y SCULLY, 1995) hasta los estudios más recientes (PAYNE et al., 2006; JONES, 2005 y 2007; KITSCHELT et al., 2010), califica, sin dejar espacio para dudas, al sistema de partidos uruguayo como altamente institucionalizado. La noción de institucionalización de los sistemas de partidos, que ya podríamos calificar como clásica, está fuertemente asociada a la idea de estabilidad, especialmente en el terreno electoral. Sin embargo, el sistema de partidos uruguayo ha sufrido transformaciones muy significativas a lo largo de las últimas décadas. En este trabajo se asume que la institucionalización de un sistema de partidos es un proceso de largo plazo y que para comprenderlo se requiere observar con cierto detalle la evolución histórico-política del sistema y de sus integrantes.

La institucionalización de un sistema de partidos es el resultado de procesos de acumulación y aprendizajes, tanto por parte de las elites políticas cuanto de la ciudadanía. Los sistemas de partidos no se institucionalizan de un día para otro y, por lo tanto, la explicación del resultado (la institucionalización) debe ser subsidiaria de la 
evolución en el mediano y largo plazo de la acción de élites y votantes de los respectivos sistemas políticos. La institucionalización de los sistemas de partidos no se manifiesta en la mera continuidad in eternum de sus partidos políticos. En palabras de Cavarozzi y Casullo "la experiencia latinoamericana nos enseña que la institucionalización política sería, en su grado óptimo, una delicada dinámica homeostática, más que una simple posición estática del sistema” (2002, p. 25). En otras palabras, los sistemas institucionalizados no serían simplemente aquellos que ofrecen continuidad sino, especialmente, los que son capaces de procesar una transformación cuando esta es requerida sin que se produzca una crisis o una ruptura institucional.

Precisamente, un análisis detallado de la evolución del sistema de partidos uruguayo permitirá mostrar que lo que lo ha caracterizado durante las últimas décadas no es la mera estabilidad, sino una peculiar forma de cambio que se desarrolla de forma gradual en el contexto de importantes continuidades. En Uruguay se ha procesado una lenta transformación del sistema partidario que culminó con el triunfo electoral del Frente Amplio en 2004. Precisamente, la capacidad de los sistemas de partidos de incorporar nuevos actores que logren integrarse de forma exitosa podría ser el rasgo decisivo que determina su nivel de institucionalización (CARIBONI LÓPEZ, 2005). En definitiva, la institucionalización de un sistema de partidos no parece estar determinada por la continuidad de sus componentes sino por la continuidad de las pautas de interacción entre componentes, que pueden cambiar a lo largo del tiempo.

En la actualidad el sistema de partidos uruguayo se caracteriza por la presencia de cuatro partidos con representación parlamentaria. Los dos partidos tradicionales, PC y PN, son tan antiguos como el país mismo y, a lo largo de su historia, prácticamente siempre han ocupado posiciones de gobierno a nivel nacional y municipal. Hasta la década de 1960 ambos partidos configuraron un esquema bipartidista que desapareció en 1971 al formarse el FA a partir de un núcleo de partidos menores, llamados "de ideas" . Adicionalmente existe un cuarto partido con representación parlamentaria de escasa magnitud, el Partido Independiente (PI), que es producto de una escisión del FA y se identifica con la centroizquierda.

\footnotetext{
${ }^{2}$ Los "partidos de ideas" eran la Unión Cívica del Uruguay (católicos), el Partidos Socialista y el Partido Comunista. En la década de 1960 la Unión Cívica tomó posiciones de izquierda, adoptó la denominación de Partido Demócrata Cristiano y coincidió con socialistas y comunistas en la formación del FA.
} 
Gráfico 1 - Evolución electoral en Uruguay (1971-2009)

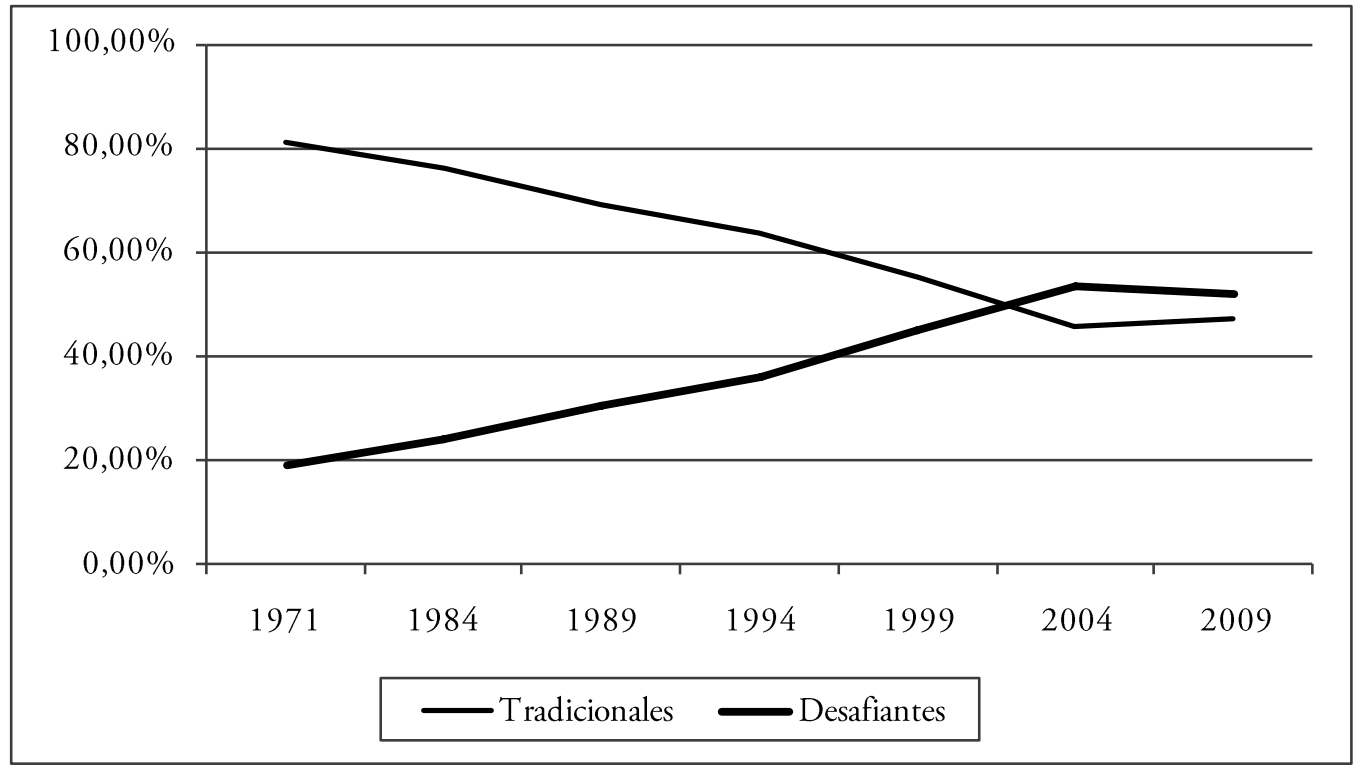

Fuente: Elaboración propia a partir de datos de la FCS-UdelaR.

El sistema de partidos uruguayo ha venido sufriendo una progresiva transformación en las últimas décadas a partir de una tendencia que comenzó a manifestarse en 1971 y que se fue consolidando con el correr de los años. Los cambios han consistido en la sistemática pérdida de votos de los partidos tradicionales y el consecuente crecimiento electoral de los "partidos desafiantes" (GONZÁLEZ y QUEIROLO, 2000), que culmina con el triunfo del FA de 2004 (Gráfico 1). Ese proceso ha sido abundantemente explicado por la academia a partir de factores económicos (crisis estructural), sociales (renovación del electorado y características socio-económicas de la población), y políticos (moderación ideológica del FA vs. derechización de los partidos tradicionales) ${ }^{3}$.

El reflejo de este proceso de cambio electoral en la dinámica de gobierno condujo al sistema político uruguayo post bipartidista a exhibir rasgos de la llamada "combinación difícil”“. La distribución de fuerzas políticas en el Uruguay desde 1971 marcaba la imposibilidad crónica de que el gobierno electo contara con mayorías

\footnotetext{
${ }^{3}$ La literatura sobre el tema es abrumadora. Para tener un panorama de la elaboración más reciente de la academia uruguaya se pueden consultar las compilaciones de Lanzaro (2004) y Buquet (2005).

${ }^{4}$ Problemas típicos de un diseño institucional presidencialista que, según Mainwaring (1993) podían presentarse cuando un esquema multipartidista en el parlamento dificulta la consecución de mayorías legislativas para el gobierno.
} 
parlamentarias propias; dificultad que se magnifica si se considera que el Presidente de la República no suele ser el líder indiscutido de su partido sino tan sólo de su fracción mayoritaria.

Tabla 1 - Evolución de la composición del Parlamento uruguayo (1984-2009)

\begin{tabular}{|c|c|c|c|c|c|c|c|}
\hline & & 1984 & 1989 & 1994 & 1999 & 2004 & 2009 \\
\hline \multirow{2}{*}{ Frente Amplio } & Senadores & $\begin{array}{c}6 \\
(19,4 \%)\end{array}$ & $\begin{array}{c}7 \\
(22,6 \%)\end{array}$ & $\begin{array}{c}9 \\
(29,0 \%)\end{array}$ & $\begin{array}{c}12 \\
(38,7 \%)\end{array}$ & $\begin{array}{c}17 \\
(54,8 \%)\end{array}$ & $\begin{array}{c}17 \\
(54,8 \%)\end{array}$ \\
\hline & Diputados & $\begin{array}{c}21 \\
(21,2 \%)\end{array}$ & $\begin{array}{c}21 \\
(21,2 \%)\end{array}$ & $\begin{array}{c}31 \\
(31,3 \%)\end{array}$ & $\begin{array}{c}40 \\
(40,4 \%)\end{array}$ & $\begin{array}{c}52 \\
(52,5 \%)\end{array}$ & $\begin{array}{c}50 \\
(50,5 \%)\end{array}$ \\
\hline \multirow{2}{*}{$\begin{array}{c}\text { Partido } \\
\text { Nacional }\end{array}$} & Senadores & $\begin{array}{c}11 \\
(35,5 \%)\end{array}$ & $\begin{array}{c}13 \\
(41,9 \%)\end{array}$ & $\begin{array}{c}10 \\
(32,3 \%)\end{array}$ & $\begin{array}{c}7 \\
(22,6 \%)\end{array}$ & $\begin{array}{c}11 \\
(35,5 \%)\end{array}$ & $\begin{array}{c}9 \\
(29,0 \%)\end{array}$ \\
\hline & Diputados & $\begin{array}{c}35 \\
(35,4 \%)\end{array}$ & $\begin{array}{c}39 \\
(39,4 \%)\end{array}$ & $\begin{array}{c}31 \\
(31,3 \%)\end{array}$ & $\begin{array}{c}22 \\
(22,2 \%) \\
\end{array}$ & $\begin{array}{c}36 \\
(36,4 \%) \\
\end{array}$ & $\begin{array}{c}30 \\
(30,3 \%) \\
\end{array}$ \\
\hline \multirow{2}{*}{$\begin{array}{c}\text { Partido } \\
\text { Colorado }\end{array}$} & Senadores & $\begin{array}{c}14 \\
(45,2 \%)\end{array}$ & $\begin{array}{c}9 \\
(29,0 \%)\end{array}$ & $\begin{array}{c}11 \\
(35,5 \%)\end{array}$ & $\begin{array}{c}11 \\
(35,5 \%)\end{array}$ & $\begin{array}{c}3 \\
(9,7 \%)\end{array}$ & $\begin{array}{c}5 \\
(16,1 \%)\end{array}$ \\
\hline & Diputados & $\begin{array}{c}41 \\
(41,4 \%)\end{array}$ & $\begin{array}{c}30 \\
(30,3 \%)\end{array}$ & $\begin{array}{c}32 \\
(32,3 \%)\end{array}$ & $\begin{array}{c}33 \\
(33,3 \%)\end{array}$ & $\begin{array}{c}10 \\
(10,1 \%)\end{array}$ & $\begin{array}{c}17 \\
(17,2 \%)\end{array}$ \\
\hline \multirow{2}{*}{ Otros } & Senadores & $\begin{array}{c}0 \\
(0 \%)\end{array}$ & $\begin{array}{c}2 \\
(29,0 \%)\end{array}$ & $\begin{array}{c}1 \\
(3,2 \%)\end{array}$ & $\begin{array}{c}1 \\
(3,2 \%)\end{array}$ & $\begin{array}{c}0 \\
(0 \%)\end{array}$ & $\begin{array}{c}0 \\
(0 \%)\end{array}$ \\
\hline & Diputados & $\begin{array}{c}2 \\
(2,0 \%)\end{array}$ & $\begin{array}{c}9 \\
(9,1 \%)\end{array}$ & $\begin{array}{c}5 \\
(5,1 \%)\end{array}$ & $\begin{array}{c}4 \\
(4,0 \%)\end{array}$ & $\begin{array}{c}1 \\
(1,0 \%)\end{array}$ & $\begin{array}{c}2 \\
(2,0 \%)\end{array}$ \\
\hline
\end{tabular}

Fuente: Elaboración propia a partir de datos de la FCS-UdelaR.

Este factor es el que ha hecho que desde el gobierno de Juan María Bordaberry (1972-1973) en adelante, el gabinete ministerial siempre contara con integrantes extrapartidarios a cambio de los necesarios votos que requería la conformación de una mayoría en el parlamento 5 . Desde la "entonación nacional" de Julio María Sanguinetti (1985-1990), pasando por la "coincidencia nacional" de Luis Alberto Lacalle (1990-1995), hasta las coaliciones explícitas entre colorados y blancos de la segunda administración Sanguinetti (1995-2000) y la de Jorge Batlle (20002005), que por primera vez incluyeron a la totalidad de los dos partidos tradicionales, existió una misma realidad subyacente: el partido que accedía al gobierno no contaba con mayorías legislativas propias y debía pasar por la formación de acuerdos políticos más o menos estables con otros partidos - es decir coaliciones - para poner en práctica su programa de gobierno. Estos rasgos formaron parte del sustento argumental de la reforma constitucional de 1997 que introdujo - entre otras cosas -

\footnotetext{
${ }^{5}$ Chasquetti (1998) analiza la formación de coaliciones en Uruguay en sus diversas modalidades.
} 
el balotaje para la elección presidencial sobre la base de que tal instrumento facilitaría la realización de acuerdos políticos interpartidarios.

La reforma de 1997 vino a conformar el marco institucional que se adecuó y consolidó una dinámica política de bloques diferenciados ideológicamente. De un lado el FA fue ocupando crecientemente el espacio desde la izquierda hacia el centro del espectro ideológico, al tiempo que los partidos tradicionales se fueron restringiendo a la zona del centro a la derecha. El FA se fue desplazando desde sus posiciones radicales originales hasta ubicarse como un partido de corte socialdemocrático ocupando un espacio que los partidos tradicionales iban dejando vacío una vez que asumían e intentaban implementar las políticas de ajuste y las reformas estructurales que se proponían en el llamdo "consenso de Washington" (LANZARO, 2004). Así la competencia política del Uruguay posdictadura se fue estructurando crecientemente en torno al eje izquierda derecha.

Los estudios de opinión pública muestran que el electorado uruguayo se distribuye ideológicamente de acuerdo a una curva "normal" y que la autoidentificación ideológica está fuertemente asociada a la intención de voto (CANZANI, 2010). Así los votantes de izquierda votan casi exclusivamente por el FA, los de derecha se distribuyen entre los partidos tradicionales y los de centro se dividen entre ambos bloques. Las elites políticas muestran también una clara diferenciación ideológica consistente con las percepciones ciudadanas. Es así que Uruguay constituye uno de los sistemas políticos de América Latina que exhibe mayores niveles de polarización ideológica ${ }^{6}$. Sin embargo, esa polarización no significa que la política uruguaya presente altos niveles de conflicto, sino más bien que el comportamiento electoral tiene fundamentos programáticos más que clientelísticos o personalistas (MAINWARING y TORCAL, 2005; KITSCHELT et al., 2010; ALTMAN et al., 2009; LUPU, 2014). Asimismo, los niveles de identificación partidaria son significativamente altos en Uruguay. Como muestra Lupu (2014), en base a datos de LAPOP, Uruguay presenta de los mayores niveles de identificación partidaria en América Latina, solo superado por los Estados Unidos y República Dominicana. En particular, Uruguay es un caso que combina alta identificación partidaria y polarización.

Pero más allá de sus características y los enfoques utilizados para su análisis, el proceso de cambio político en Uruguay había mostrado tal grado de estabilidad que

\footnotetext{
${ }^{6}$ Ver por ejemplo Jones (2005) o los datos de Encuesta de Elites Políticas de América Latina que conduce la Universidad de Salamanca, disponibles de forma agregada en $<$ http://americo.usal.es/oir/opal/indicadores.htm>.
} 
hasta una simple regresión lineal permitió predecir en el año 2000 el resultado de la elección de 2004 con un extraordinario nivel de precisión (GONZÁLEZ y QUEIROLO, 2000). Sin embargo, una vez que el FA llegó al gobierno, ese fenómeno dejó de producirse por completo, tanto el proceso de crecimiento del bloque desafiante a costa del bloque tradicional, cuanto la condición minoritaria de los presidentes electos. El proceso culminó con la consolidación de los dos bloques como tales que parecen haberse estabilizado ocupando cada uno de ellos aproximadamente la mitad del espectro político.

El sistema de partidos era estable en Uruguay hasta que el viejo equilibrio bipartidista dejó de funcionar una vez que el número de competidores comenzó a crecer, las posiciones de los partidos a variar y las preferencias de los electores a cambiar significativamente de una elección a otra. Las nuevas reglas electorales aprobadas en 1997 pretendieron adaptarse a la nueva configuración partidaria. El sistema de DV facilitó la coordinación entre blancos y colorados y alimentó la competencia entre bloques diferenciados ideológicamente. Pero las nuevas reglas también contribuyeron a que el FA pudiera resolver sus conflictos internos apelando a la competencia electoral abierta a través de las elecciones internas. Así como el sistema electoral de MR con DVS era funcional al viejo bipartidismo fraccionalizado, el actual sistema de balotaje con elecciones primarias es funcional al esquema actual de dos bloques diferenciados ideológicamente, con más de dos partidos relevantes que se estructuran en torno a dos macro fracciones internas.

A continuación se analizará la forma en que la nueva normativa electoral resulta funcional y estimula el mantenimiento de esta nueva configuración políticopartidaria.

\section{Las elecciones primarias presidenciales}

La reforma electoral de 1997 obligó a los partidos a presentar una candidatura única para Presidente y Vicepresidente, terminando de esa forma con el doble voto simultáneo (DVS) para la elección presidencial ${ }^{7}$. La eliminación de un sistema tan particular como el DVS para la elección presidencial supuso la incorporación de un mecanismo no menos original para seleccionar en cada partido la candidatura única a Presidente y Vicepresidente: elecciones primarias abiertas y simultáneas para todos los partidos. La peculiaridad del nuevo sistema estribó en que los partidos pasaran a estar

\footnotetext{
7 El DVS permitía a los electores votar por un partido y luego seleccionar una de las fórmulas presidenciales que ese partido presentaba. Resultaba electa la fórmula más votada dentro del partido más votado. En la práctica, este sistema hacía que en un mismo acto electoral se realizaran elecciones primarias o internas y la elección presidencial.
} 
obligados a realizar estas elecciones de manera simultánea cuatro meses antes de la elección nacional ${ }^{8}$.

En estas elecciones resultan designados como candidatos presidenciales dentro de cada partido aquellos precandidatos que obtengan la mayoría absoluta de los votos dentro de su partido, o más del $40 \%$ y al menos 10 puntos porcentuales de diferencia con quien quede en segundo lugar. Si ningún candidato alcanza alguno de estos dos requisitos, la nominación queda en manos de un colegio elector nacional elegido en la misma instancia.

Aunque el principal objetivo de las elecciones internas consiste en seleccionar a los candidatos presidenciales de los partidos, también cumplen diversas funciones. En el plano interpartidario, aunque allí nada está en juego formalmente, aparece como relevante contar con niveles altos de participación. Las elecciones internas se realizan con voto voluntario por lo que la concurrencia depende de la capacidad de los partidos para movilizar a los electores. Una participación alta en la interna de un partido se interpreta como una señal auspiciosa en la perspectiva de la competencia presidencial nacional.

Los efectos de las primarias abiertas han sido estudiados profusamente por quienes analizan la política estadounidense. Algunos trabajos sugieren que la realización de primarias abiertas pueden generar efectos perjudiciales para los partidos políticos. En el caso de las elecciones primarias en Estados Unidos, la evidencia muestra que los partidos que tienen una contienda interna más reñida suelen ser perjudicados en las elecciones generales (ATKESON, 1998). Cuando enfrentan una competencia ajustada, los precandidatos tienden a elevar el nivel de crítica hacia su o sus rivales $\mathrm{y}$, consecuentemente, a recibir también críticas más duras. El candidato triunfante en una instancia con esas características sale debilitado de la competencia interna al tiempo que su rival en la elección nacional tiene a su disposición las críticas que el rival interno utilizó en su momento.

La evidencia que surge de la realización de elecciones primarias en Uruguay es escasa (las elecciones internas sólo se han celebrado en tres ocasiones y sólo tres partidos han presentando competencia por la candidatura presidencial en esa instancia) y no parece concluyente en ese sentido. Si bien en una ocasión (2004) el ganador de la elección nacional (Vázquez - FA) no tuvo rivales en la primaria, en las

\footnotetext{
${ }^{8}$ El artículo $8^{\circ}$ de la Ley 17.063 - que dicta normas relativas a las elecciones internas de los partidos establece que: "Las elecciones internas referidas en la Disposición Transitoria W) de la Constitución de la República se realizarán en un único acto, con sufragio secreto y no obligatorio, en el que en una hoja de votación, identificada por el lema partidario, se expresará el voto por el candidato único del partido político a la Presidencia de la República...”.
} 
otras dos ocasiones (1999 y 2009) los triunfadores en la elección nacional (Batlle PC y Mujica - FA) obtuvieron su candidatura a través de la contienda más competitiva de todas, es decir en la que se verifica la menor diferencia entre los dos primeros competidores (ver Tabla 2).

Tabla 2 - Votación de los dos primeros candidatos, diferencia y número efectivo de candidatos en las primarias de 1999, 2004 y 2009

\begin{tabular}{|c|c|c|c|c|c|c|c|}
\hline & & & 1999 & & 2004 & & 2009 \\
\hline \multirow{4}{*}{$\begin{array}{c}\text { Partido } \\
\text { Colorado }\end{array}$} & $1^{\circ}$ & Batlle & $54,92 \%$ & Stirling & $91 \%$ & Bordaberry & $72,2 \%$ \\
\hline & $2^{\circ}$ & Hierro & $44,14 \%$ & Iglesias & $7 \%$ & Amorín & $14,7 \%$ \\
\hline & Diferencia & & $10,8 \%$ & & $84,2 \%$ & & $57,5 \%$ \\
\hline & $\mathrm{NEC}^{*}$ & & 2,01 & & 1,2 & & 1,8 \\
\hline \multirow{4}{*}{$\begin{array}{c}\text { Partido } \\
\text { Nacional }\end{array}$} & $1^{\circ}$ & Lacalle & $48,3 \%$ & Lacalle & $34 \%$ & Lacalle & $57,1 \%$ \\
\hline & $2^{\circ}$ & Ramírez & $32,3 \%$ & Larrańaga & $66 \%$ & Larrańaga & $42,8 \%$ \\
\hline & Diferencia & & $16,0 \%$ & & $33 \%$ & & $14,3 \%$ \\
\hline & \begin{tabular}{|l|} 
NEC $^{*}$ \\
\end{tabular} & & 2,8 & & 1,8 & & 2,0 \\
\hline \multirow{4}{*}{$\begin{array}{l}\text { Frente } \\
\text { Amplio }\end{array}$} & $1^{\circ}$ & Vázquez & $82,4 \%$ & Vázquez & $100 \%$ & Mujica & $52,0 \%$ \\
\hline & $2^{\circ}$ & Astori & $17,6 \%$ & & & Astori & $39,7 \%$ \\
\hline & Diferencia & & $64,8 \%$ & & $100 \%$ & & $12,4 \%$ \\
\hline & $\mathrm{NEC}^{*}$ & & 1,4 & & 1 & & 2,3 \\
\hline
\end{tabular}

* Número efectivo de candidatos de acuerdo a la fórmula de Laasko y Taagepera 1979. Fuente: Elaboración propia a partir de datos de la FCS-UdelaR.

Más allá de que la experiencia uruguaya no permita obtener conclusiones contundentes sobre los efectos de la competencia primaria en el desempeño de los partidos en la elección general, pueden apuntarse algunas tendencias observadas a lo largo de la última década. En primer término las elecciones primarias han estimulado su utilización efectiva para designar el candidato presidencial de los partidos principales. Si bien esto puede resultar natural para los partidos tradicionales que siempre habían utilizado el DVS con esa finalidad, no es así en el caso del FA que, al contrario, siempre criticó la multiplicidad de candidaturas presidenciales. La obligatoriedad de las elecciones internas, sumada a los procesos de pugna interna en el FA condujeron finalmente a que este partido también haya resuelto definir su candidato presidencial en una competencia interna abierta, en una decisión que parece no tener retorno mientras exista más de un aspirante a la nominación.

Por otra parte, la naturaleza mayoritaria de la elección primaria (más allá de los umbrales establecidos) genera incentivos para que la competencia se concentre entre dos competidores principales. La evolución del número efectivo de candidatos (NEC), si bien se redujo para todos los partidos en 2004 con respecto a 1999, 
terminó ubicándose en valores aproximados a 2 para todos los partidos en 2009. En este sentido, la elección primaria refuerza el esquema bifraccional que históricamente han mostrado los partidos tradicionales e impulsa al FA en la misma dirección (BUQUET, CHASQUETTI y MORAES, 1998).

Finalmente, también puede apreciarse una regularidad estratégica: los partidos con internas competitivas entre dos candidatos principales tienden a armar su fórmula presidencial con el ganador como presidente y el segundo como vicepresidente (Batlle-Hierro en 1999 y Lacalle-Larrañaga y Mujica-Astori en 2009). Este fenómeno ocurrió en todos los casos en que la diferencia entre el primero y el segundo (ver Tabla 2) fue inferior a 15 puntos porcentuales y siempre que se utilizó resultó ganadora de la presidencia una fórmula con esas características. Evidentemente esta estrategia pretende compensar el efecto perjudicial mencionado más arriba que la reñida competencia interna pueda tener en el desempeño del partido en la elección general. Asimismo, parece utilizarse como un recurso para moderar la oferta presidencial de los partidos cuando el ganador de la interna, como en 2009 en el PN y el FA, es el candidato que el electorado percibe como más extremista entre los que compitieron en la primaria (BUQUET y PIŃEIRO, 2011a).

La definición de las fórmulas presidenciales en los principales partidos muestra que si bien las primarias sirven para saldar conflictivos procesos de selección de candidaturas candidaturas (KEMAHLIOGLU, WEITZ-SHAPIRO y HIRANO, 2009), no los eliminan por completo. La competencia interna, cuando es ajustada, tiende a estimular a los candidatos a profundizar en sus diferencias y luego hace difícil recomponer una oferta que contemple a las principales corrientes del partido y elimine fugas de potenciales electores cercanos a los candidatos perdedores. En ese sentido, la fórmula presidencial con los dos principales competidores de la interna busca reunificar al partido detrás del candidato triunfador. Pero, a su vez, como la realización de primarias masivas también favorece el triunfo de los candidatos más radicales en cada uno de los partidos (COLOMER, 2002; BUQUET y CHASQUETTI, 2008; BUQUET y PINEEIRO, 2011a) el principal objetivo en la elección de los candidatos a vicepresidente fue el de complementar el perfil de los candidatos a presidente con las principales figuras de los sectores más moderados?

\footnotetext{
${ }^{9}$ Por lo menos en los dos partidos mayores de la elección de 2009, resulta innegable que tanto Mujica como Lacalle se ubicaban más distantes del centro ideológico que sus respectivos rivales internos (BUQUET y PIŃEIRO, 2011a).
} 


\section{Elecciones nacionales y alternancia}

En 2004 el FA ganó la Presidencia de la República y una mayoría absoluta en el Poder Legislativo, con el PN ubicándose como principal partido de oposición y el PC relegado a un lejano tercer lugar. En 2009 ocurrió prácticamente lo mismo; he ahí la gran novedad. El principal cambio que ha experimentando el sistema de partidos uruguayo en el pasado ciclo electoral es que ha dejado de cambiar. La "normalización" que representa la elección pasada puede apreciarse observando la evolución de la volatilidad electoral ocurrida en nuestro país a lo largo de las últimas siete décadas.

Gráfico 2 - Volatilidad electoral en Uruguay (1946-2009)

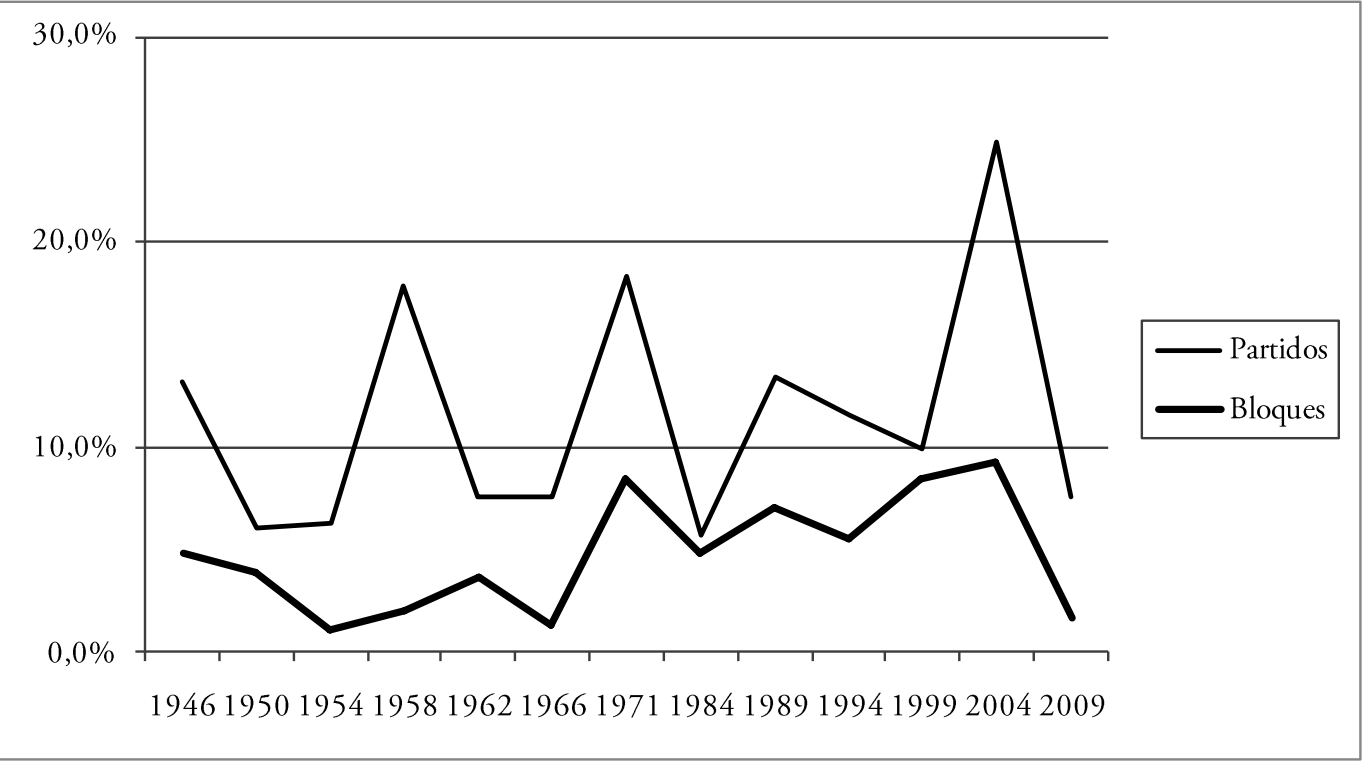

Fuente: Elaboración propia a partir de datos de la FCS-UdelaR.

La volatilidad representa el cambio "neto" de votos entre partidos o bloques de una elección a otra ${ }^{10}$. Uruguay es un país que presenta valores moderados en este indicador y los picos de volatilidad entre partidos que se aprecian en el Gráfico 2 corresponden a "elecciones críticas"11: el triunfo del PN de 1958 luego de décadas de

\footnotetext{
${ }^{10}$ Para calcular la volatilidad normalmente se utiliza el índice de Pedersen (1979), que se calcula como la semisuma de las diferencias porcentuales obtenidas por cada partido o bloque entre dos elecciones.

${ }^{11}$ Burnham (1970), en su libro Critical Elections and the Mainsprings of American Politics, introdujo el concepto de "elecciones críticas". Con ello apuntó a la existencia de un fenómeno mediante el cual el electorado, cada cierto período de tiempo, experimenta un realineamiento mayor que tiende a predominar en el mediano plazo.
} 
hegemonía colorada, la formación del FA en 1971 y su triunfo en 2004. A su vez, la volatilidad entre bloques hasta 1971 era muy poco significativa ya que las principales transferencias de votos se producían entre los partidos tradicionales. En cambio, la volatilidad entre bloques a partir de 1971 se incrementó sensiblemente, llegando por momentos a ser casi igual a la volatilidad total, porque en ese periodo los principales cambios de votos ocurrían desde un bloque al otro. Es decir que en el tramo que va desde 1971 hasta 2004 el principal cambio electoral en Uruguay se manifestó en un flujo más o menos permanente de votos desde los partidos tradicionales hacia el FA.

La volatilidad que arrojó la última elección nacional se redujo significativamente con respecto a la anterior y los valores correspondientes se ubican entre los menores de toda la serie, muy por debajo del promedio. La volatilidad entre partidos en la elección de 2009 se situó en un 7,6\%, casi cuatro puntos por debajo del promedio de toda la serie que es de $11,5 \%$. Por su parte, la volatilidad entre bloques se ubicó en $1,7 \%$, valor que representa poco más de la tercera parte del promedio de la serie $(4,8 \%)$. En consecuencia nos encontramos efectivamente frente a un proceso electoral que ofreció muy poco cambio, al menos en los aspectos cuantitativos de la competencia entre partidos.

En particular, es importante subrayar el bajo nivel de la volatilidad entre bloques porque es la que está asociada al proceso de largo plazo que se mencionó antes, ya que mide la transferencia neta de votos entre los partidos tradicionales y la izquierda. En ese sentido el valor de la última elección implica un cambio sustancial con referencia al periodo 1971-2004, donde el guarismo más bajo se ubicó prácticamente en el triple de esa cifra $(4,9 \%)$. En realidad, la volatilidad entre bloques de 2009 es incluso baja para el periodo bipartidista de 1946 a 1966, cuyo promedio es de 2,9\%. Concretamente, si desagregamos la serie en esos dos periodos, los valores de volatilidad de 2009 se parecen más a los del Uruguay bipartidista que a los del periodo posterior dentro del que se produjeron los cambios en el sistema de partidos. En definitiva, la elección de 2009 tiene las características de las que se realizan dentro de un periodo de estabilidad en la configuración del sistema de partidos y no de las que ocurren en un periodo de transformaciones. El análisis de la evolución de estos indicadores reafirma la idea de que el sistema de partidos uruguayo habría alcanzado un nuevo equilibrio competitivo en el último proceso electoral.

La idea de un equilibrio competitivo implica la presencia de una determinada configuración partidaria y un conjunto de reglas que generan incentivos para que esa configuración se mantenga estable. En otras palabras, dadas las reglas, los actores políticos no tienen incentivos para modificar sus estrategias. Las elites políticas deciden mantener en términos similares la oferta electoral (los partidos y los 
candidatos) y los electores mantienen en términos generales sus preferencias. Los cambios que ocurren son menores y derivan fundamentalmente de los desempeños políticos en el corto plazo.

En Uruguay se utilizó hasta 1994 el sistema de mayoría relativa (MR) con DVS para la elección presidencial y, a partir de 1999, el sistema de doble vuelta o balotaje unido a elecciones primarias. En ese sentido, el sistema anterior se asociaba a un sistema bipartidista $^{12}$. Incorporando los efectos del DVS, la literatura académica uruguaya coincidió al explicar la permanencia durante varias décadas de un sistema bipartidista fraccionalizado en nuestro país como una consecuencia del sistema electoral (PÉREZ, 1970; GONZÁLEZ, 1993; BUQUET, CHASQUETTI y MORAES, 1998). En consecuencia se puede decir que el Uruguay bipartidista con su viejo sistema electoral se encontraba en equilibrio.

Gráfico 3 - Evolución de la fragmentación partidaria en Uruguay (1946-2009) Número efectivo de partidos (electoral)

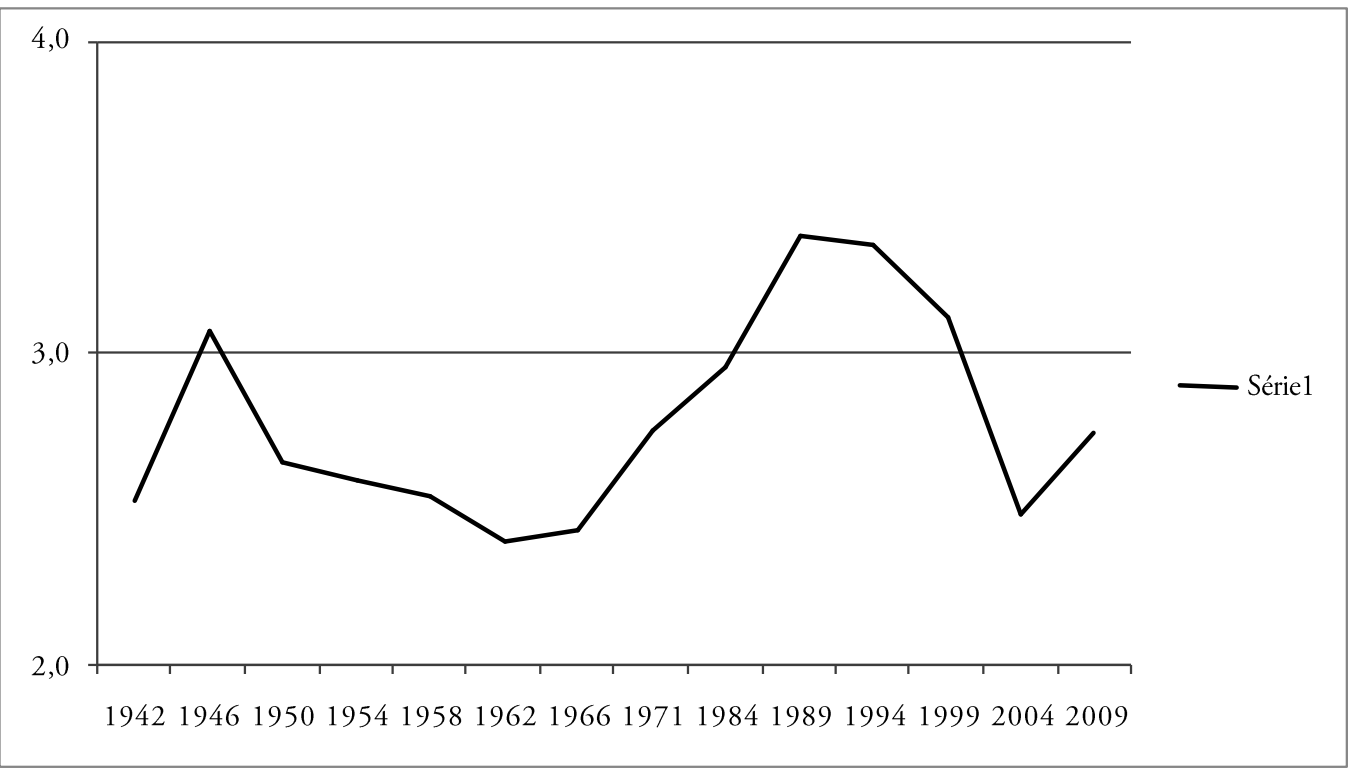

Fuente: Elaboración propia a partir de datos de la FCS-UdelaR.

Por su parte, el uso del sistema de doble vuelta (DV), adoptado en Uruguay como consecuencia del crecimiento del FA, debería asociarse a tres competidores viables, ya que la elección en primera vuelta genera potencialmente dos ganadores que

\footnotetext{
${ }^{12}$ De acuerdo con la ley de Duverger (1957) que sostiene que los sistemas de mayoría relativa promueven el bipartidismo.
} 
son los que pasan a competir en la segunda vuelta (COX, 1997). En el terreno empírico, se ha podido verificar que los sistemas de elección de DV se asocian en América Latina a sistemas partidarios más fragmentados que los de MR (SHUGART y CAREY, 1992; MAINWARING y SHUGART, 1997). En consecuencia, debería esperarse que la incorporación del sistema de DV en Uruguay favoreciera la fragmentación del sistema de partidos, a diferencia del sistema de MR que favorece la concentración. Sin embargo la evolución del número efectivo de partidos ${ }^{13}$ (NEP) no mostró una tendencia al aumento de la fragmentación en este periodo.

El balotaje fue implementado luego de que el sistema de partidos había llegado al punto de mayor fragmentación $(\mathrm{NEP}=3,4)$ y comenzaba su reducción (Gráfico 3). Esa reducción continuó hasta la elección de 2004, a pesar de que la regla de DV ya no generaba incentivos estratégicos para la concentración electoral. Finalmente, la última elección de la serie muestra que por primera vez en 20 años el NEP vuelve a incrementarse. Esta evolución parece contradecir las leyes de Duverger; mientras regía un sistema de MR el sistema tendió a fragmentarse y a partir de que se adoptó la DV el sistema tendió a concentrarse. Sin embargo, este proceso puede verse de una forma diferente. El cumplimiento de las leyes de Duverger depende de los comportamientos estratégicos de partidos y electores ${ }^{14}$. Pero esos comportamientos estratégicos no tienen por qué ocurrir como una respuesta automática a la regla sino más bien como consecuencia de un aprendizaje que se realiza a lo largo del tiempo en una secuencia de aciertos y errores. En ese sentido es posible suponer que una vez que se adoptó el balotaje los comportamientos de los electores mantuvieron una inercia, que correspondía a los incentivos generados por el sistema anterior, y que recién en la última elección comenzaron a responder a los incentivos del sistema de DV. Si esto fuera cierto sería esperable que en el futuro vuelva a incrementarse la fragmentación continuando la tendencia iniciada en la elección de 2009 y se mantenga en torno a tres partidos tal como predice la teoría.

\section{Elecciones subnacionales y fragmentación}

En Uruguay se eligen simultáneamente 19 gobiernos subnacionales que corresponden a los departamentos en los que está dividida administrativamente la república. En esa instancia se dirimen el cargo de Intendente Departamental, que corresponde al ejecutivo local y los 31 escaños de la Junta Departamental, un ámbito deliberativo y legislativo. Desde el año 2000 esas elecciones se realizan el año

\footnotetext{
13 El número efectivo de partidos es un índice propuesto por Laakso y Taagepera (1979) para establecer el número de partidos relevantes que compiten en un sistema.

${ }^{14}$ Lo que Duverger (1957) denominaba "efecto psicológico".
} 
siguiente a las elecciones nacionales. El sistema de elección de intendentes, desde la reforma constitucional de 1997 difiere del sistema de elección presidencial en tres aspectos relevantes: i) la fórmula para elegir al intendente es de mayoría relativa, en lugar de la mayoría absoluta que se utiliza para elegir al presidente; ii) los partidos pueden presentar hasta tres candidatos a intendente, a diferencia del candidato único que se exige a los partidos en las elecciones presidenciales; y iii) está permitida la reelección inmediata de los intendentes por una vez, mientras que los presidentes que aspiran a retornar al cargo deben esperar que se cumpla un periodo de gobierno.

Cada una de estas diferencias genera potencialmente impactos en los resultados y, por tanto, incentiva diversos comportamientos estratégicos en políticos y ciudadanos. Tanto la mayoría relativa como la presencia de intendentes que aspiran a la reelección, son factores que promueven la concentración de la competencia electoral (COX, 1997; JONES, 1999). Aunque los datos de elecciones nacionales no permiten confirmar que el sistema de DV promueve la fragmentación, porque el número de observaciones es muy reducido y la evolución de la fragmentación nacional no muestra una tendencia en ese sentio, se puede comprobar lo contrario utilizando los datos a nivel departamental: que el uso del sistema de MR para la elección de intendentes está asociado a una reducción de la fragmentación. Las elecciones departamentales se realizan siete meses después que las elecciones nacionales y en ellas votan prácticamente los mismos electores y compiten los mismos partidos. En esta situación los comportamientos estratégicos de los electores (cuando no existe coordinación previa por parte de las elites) son claramente esperables, no sólo porque el método de MR los estimula, sino porque los electores cuentan con información precisa, proporcionada por el resultado departamental de la elección nacional anterior, que les permite saber qué partidos cuentan con chance de competir con la intendencia.

Para verificar que el sistema de MR promueve una reducción de la fragmentación, a través de la coordinación estratégica de los votantes, se desarrolla un modelo estadístico. El modelo que se propone consiste en tomar la fragmentación electoral que se produce en cada uno de los 19 departamentos en la elección nacional para compararla con la fragmentación que se observa en las correspondientes elecciones departamentales. De esa forma es posible asumir que utilizamos un diseño cuasi experimental que expone a los mismos electores ante dos elecciones que se dirimen por reglas diferentes (BUQUET, 2004). La hipótesis es que el NEP departamental en las elecciones de Intendente, que se rigen por MR debe ser significativamente inferior al NEP en ese mismo departamento en las elecciones presidenciales anteriores realizadas por el sistema de DV. Esto es posible, además, 
porque la reforma electoral separó en el tiempo la elección departamental de la nacional permitiendo independizar la decisión de voto entre una y otra instancia. De esta forma contamos con 114 observaciones (19 votaciones nacionales y 19 departamentales para cada uno de los tres ciclos electorales ocurridos después de la reforma: 1999-2000, 2004-2005 y 2009-2010), cantidad suficiente para un tratamiento estadístico de la información.

El modelo además incluye una serie de variables de control. En primer término parece relevante considerar que en la elección de intendente, a diferencia de la presidencial, se admite la reelección inmediata por una vez. Se ha mencionado que la presencia del ocupante del cargo (incumbent) como candidato a volver a ocuparlo podría generar por sí misma un efecto reductor en la competencia (JONES, 1999) aunque la evidencia empírica no es contundente en ese sentido (CARDARELLO, 2009). Por lo tanto, el modelo incluye una variable (dummy) para indicar la presencia de un intendente que aspira a la reelección. Por otra parte, tanto características de la coyuntura política, como particularidades de los departamentos podrían tener efectos sobre el comportamiento electoral. Con relación a la coyuntura política incluimos dos variables (2004-2005 y 2009-2010) para controlar por cada ciclo electoral y para controlar las particularidades locales incluimos el número efectivo de partidos en la elección departamental anterior. Esta última variable permite a su vez controlar la autocorrelación de la variable dependiente, ya que los datos son de panel (las mismas unidades en diferentes momentos) y no se puede asumir la completa independencia de las observaciones.

Tabla 3 - Resultados de la regresión sobre número efectivo de partidos

\begin{tabular}{c|c|c|c}
\hline & $\begin{array}{c}\text { Unstandardized } \\
\text { Coefficients }\end{array}$ & \multicolumn{2}{|c}{ Sig. } \\
\hline (Constant) & $\mathrm{B}$ & Std. Error & Sig \\
\hline Tipo de elección & 2,143 &, 248 &, 000 \\
\hline Intendente por reelección &,- 359 &, 074 &, 000 \\
\hline Ciclo 2004-2005 &,- 041 &, 073 &, 581 \\
\hline Ciclo 2009-2010 &,- 359 &, 061 &, 000 \\
\hline $\begin{array}{c}\text { aúmero efectivo de partidos en la elección } \\
\text { anterior }\end{array}$ &,- 074 &, 070 &, 297 \\
\hline R cuadrado ajustado &, 289 &, 081 &, 001 \\
\hline Número de casos & & &, 551 \\
\hline
\end{tabular}

Fuente: Elaboración propia. 
La Tabla 3 muestra los resultados del modelo de regresión lineal (OLS). El NEP departamental promedio en elecciones nacionales es de 2,8, un valor que se aproxima al tripartidismo, mientras que el NEP promedio de las elecciones departamentales es de 2,3, cifra que se ubica más próxima a un esquema de competencia bipartidista y la diferencia entre ambos valores es significativa estadísticamente. La variable independiente de la hipótesis es significativa, de lo que podemos deducir que la regla de elección efectivamente produce el efecto esperado, confirmando la ley de Duverger para la competencia en las elecciones departamentales uruguayas: el sistema de elección por MR favorece la concentración electoral reduciendo el número efectivo de competidores y tendiendo hacia un esquema de competencia bipartidista.

Por otra parte, el nivel de concentración electoral no es independiente de la coyuntura política, ya que la variable "ciclo 2004-2005" también es significativa estadísticamente. De todas formas esto no significa que todas las coyunturas generen efectos similares porque la variable "ciclo 2009-2010" no es estadísticamente significativa. Parece razonable pensar que ciertas coyunturas tengan impactos relevantes por sí solas; en particular el ciclo electoral de 2004-2005 se produjo luego de la peor crisis económica en la historia del país y esas elecciones arrojaron niveles récord de volatilidad electoral. Asimismo, el NEP anterior también influye en el NEP actual mostrando que el resultado de una elección en un lugar en un momento t no es independiente del resultado electoral en t-1. En definitiva, más allá de las reglas existen particularidades locales y coyunturales que inciden en los niveles de fragmentación electoral. Por último, la presencia de intendentes compitiendo por la reelección no genera efectos relevantes en la fragmentación del sistema de partidos, confirmando los hallazgos de Cardarello (2009) para el caso uruguayo.

Aunque el análisis de la información de esta sección muestra directamente sólo que el sistema de elección por MR favorece la concentración electoral, indirectamente permite inferir que el uso de la DV en la elección presidencial favorece la estabilidad de un formato más fragmentado del sistema de partidos y, por lo tanto, no ejerce una presión para modificar su configuración actual.

\section{Conclusiones}

A pesar de su alto grado de institucionalización y de la relativa estabilidad de la competencia electoral el sistema de partidos uruguayo ha sufrido un significativo cambio en el mediano plazo. En 2004 las elecciones nacionales ofrecieron un resultado que fue evaluado naturalmente como uno de los cambios políticos más relevantes en la historia del Uruguay. El acceso al gobierno del FA constituyó una 
novedad absoluta para un país que había sido gobernado sólo por sus dos partidos tradicionales a lo largo de toda su historia. En cambio, las elecciones realizadas en 2009 lucen más bien como una reproducción de aquellas. Así, la lectura dominante del ciclo electoral anterior fue realizada en clave de cambio y la del actual, con un componente sustancial de continuidad. Sin embargo, si en lugar de enfocar en quién obtiene el gobierno y en la distribución de fuerzas políticas, se pone la mirada en los procesos de mediano plazo puede decirse exactamente lo contrario: los resultados de 2004 no reflejan otra cosa que la continuidad - y la culminación - de un proceso de largo aliento, mientras que los de 2009 son verdaderamente novedosos (aunque no necesariamente sorprendentes) y muestran un cambio fundamental en la dinámica política nacional.

Lo que estamos en condiciones de afirmar sobre la elección de 2004, teniendo a la vista los resultados de 2009, es que aquella elección fue precisamente la culminación de un proceso de transformación de la configuración del sistema de partidos uruguayo, que comenzó en 1971 con la formación del FA y terminó en 2004 con su acceso al gobierno. El proceso culminó efectivamente hace cinco años porque su principal característica fue el gradual y sistemático crecimiento del voto de izquierda (o, si se quiere, del voto desafiante) en detrimento del caudal electoral de los partidos tradicionales; todas las elecciones desde 1971 hasta 2004 inclusive, mostraron en mayor o menor medida, esa particularidad. Lo que hace de la elección nacional de 2009 una novedad absoluta es que por primera vez desde entonces el FA (y el subsistema desafiante) reduce su porcentaje de votación y los partidos tradicionales sumados la incrementan.

$\mathrm{Si}$ un proceso electoral muestra muy poco cambio, podemos suponer que el sistema se encuentra en equilibrio. En ese sentido, los resultados de la elección de 2009 nos sugieren que el sistema político uruguayo encontró un nuevo equilibrio competitivo en su configuración actual y con las reglas vigentes. El sistema de DV para la elección presidencial no genera los incentivos para la concentración electoral que producía el sistema de MR. Al mismo tiempo, el sistema de primarias obligatorias ha llevado al FA a dirimir sus conflictos internos en un escenario de competencia electoral abierta que reproduce las lógicas históricas de los partidos tradicionales. Así como el sistema anterior estaba hecho a la medida del viejo sistema de partidos uruguayo, el vigente parece adecuarse cada vez más a la configuración actual. En un futuro cercano es de esperar que el sistema de partidos uruguayo se mantenga estable con tres o cuatro partidos, alineados en dos bloques diferenciados ideológicamente que dirimen sus disputas internas en elecciones primarias abiertas. El sistema político uruguayo, luego de un extenso periodo de transformaciones que 
comenzó hace más de cuatro décadas y transitó por etapas especialmente trágicas, definitivamente parece haber arribado a un nuevo equilibrio.

- Daniel Buquet é Professor e Pesquisador do Instituto de Ciência Politica e Faculdade de Ciências Sociais da Universidade da República. E-mail: danielbuquet@cienciassociales.edu.uy

- Rafael Piñeiro é Professor Assistente do Departamento de Ciências Sociais e Política da Universidade Católica do Uruguai. Email: rafael.pineiro@ucu.edu.uy

\section{Referencias}

ALTMAN, David et al. Partidos y sistemas de Partidos en América Latina: aproximaciones desde la encuesta a expertos 2009. Revista de Ciencia Politica, v. 29, n. 3, p. 775-798, 2009.

ATKESON, Lonna R. Divisive Primaries and General Election Outcomes: Another Look at Presidential Campaigns. American Journal of Political Science, v. 42 n. 1, p. 256-271, 1998.

BUQUET, Daniel. Balotaje vs. Mayoría Simple: el Experimento Uruguayo. In: MARTÍNEZ, Rafael (Ed.) La Doble Vuelta en Sudamérica, Centroamérica y Caribe. Barcelona: Institut de Ciencies Polítiques i Socials, 2004. p. $493-510$.

BUQUET, Daniel (Coord.). Las claves del cambio: Ciclo electoral y nuevo gobierno 2004-2005. Montevideo: Ediciones de la Banda Oriental, 2005.

BUQUET, Daniel; CHASQUETTI, Daniel. Presidential candidate selection in Uruguay: 1942-2004. In: SIAVELIS, Peter; MORGENSTERN, Scott (Eds.). Pathways to Power: Political Recruitment and Candidate Selection in Latin America. University Park: Penn State University Press, 2008. p. 319-359.

BUQUET, Daniel; CHASQUETTI, Daniel; MORAES, Juan A. Fragmentación Política y Gobierno en Uruguay: ¿Un enfermo imaginario? Montevideo: Instituto de Ciencia Política - Facultad de Ciencias Sociales, 1998.

BUQUET, Daniel; PIÑERO, Rafael. De las internas a las municipales: los impactos de las reglas electorales en Uruguay. In: BUQUET, Daniel; JOHNSON, Niki (Coords.). Del cambio a la continuidad: Ciclo electoral 2009-2010 en Uruguay. Montevideo: Editorial Fin de Siglo, 2010. p. 45-70.

Participación electoral en las elecciones primarias en Uruguay. Revista Debates, v. 5, n. 2, p. 79-95, 2011 a.

. Del cambio a la continuidad: las elecciones de 2009 en Uruguay. In: ALCÁNTARA, Manuel; TAGINA, María L. (Eds.). América Latina: Política y elecciones del bicentenario (20092010). Madrid: Centro de Estudios Políticos y Constitucionales, 2011b. p. 117-212.

Elecciones Uruguayas: la consolidación de un nuevo sistema de partios. In: ALCÁNTARA, Manuel; TAGINA, María L. (Coords.) Elecciones y Política en América Latina 2009 - 2011. 
México, DF: Miguel Ángel Porrúa, Instituto Federal Electoral, Cámara de Diputados y Senado de la República. 2013. p. 197-234.

BURNHAM, Walter D. Critical Elections and the Mainsprings of American Politics. New York: W. W. Norton and Co, 1970.

CANZANI, Agustín. ¿Tipos raros? La lógica de la opinión pública detrás de los resultados electorales 2009. In: BUQUET, Daniel; JOHNSON, Niki (Coords.) Del cambio a la continuidad: Ciclo electoral 2009-2010. Montevideo: Fin de Siglo, 2010. p. 135-164.

CARDARELLO, Antonio. La reelección inmediata del ejecutivo a nivel subnacional: un estudio de tres casos. 2009. 243 f. Tese (Doutorado em Ciência Política) - Programa de Pós Graduação em Ciência Política, Universidade Federal do Rio Grande do Sul, Porto Alegre, 2009.

CARIBONI LÓPEZ, Santiago. Partidos desafiantes en América Latina: representación política y estrategias de competencia de las nuevas opciones. Revista de Ciencia Politica, v. 25, n. 2, p. 37-64, 2005.

CAVAROZZI, Marcelo; CASUlLO, Esperanza. Los partidos políticos en América Latina hoy: ¿consolidación o crisis? In: CAVAROZZI, Marcelo; MEDINA, Juan M. A. (Eds.). El asedo de la política: los partidos latinoamericanos en la era neoliberal. Buenos Aires: HomoSapiens Ediciones, 2002. p. 9-30.

CHASQUETTI, Daniel. Compartiendo el Gobierno: multipartidismo y Coaliciones en Uruguay (1971-1997), Revista Uruguaya de Ciencia Politica, v. 10, p. 25-45, 1998.

. Uruguay 2008: El complejo año de las reformas. Revista de Ciencia Política, v. 28, n. 1, p. 385-403, 2008.

COLOMER, Josep M. Las Elecciones Primarias Presidenciales en América Latina y sus Consecuencias Políticas. In: CAVAROZZI, Marcelo; MEDINA, Juan M. A. (Eds.). El asedo de la política: los partidos latinoamericanos en la era neoliberal. Buenos Aires: HomoSapiens Ediciones, 2002. p. 117-134.

COX, Gary W. Making Votes Count. Strategic Coordination in the World's Electoral Systems. Cambridge: Cambridge University Press, 1997.

DUVERGER, Maurice. Los Partidos Políticos. México: Fondo de Cultura Económica, 1957.

FCS-UdelaR. Facultad de Ciencias Sociales de la Universidad de la República Oriental del Uruguay. Área de Politica y Relaciones Internacionales del Banco de Datos. Disponível em: $<$ http://www.fcs.edu.uy/seccNUA.php?tipoSecc=14>. Acesso em: 31 ago. 2013.

GONZÁLEZ, Luis E. Estructuras Politicas y Democracia en Uruguay. Montevideo: Fundación de Cultura Universitaria, 1993.

GONZÁLEZ, Luis E.; QUEIROLO, Rosario. Las elecciones nacionales de 2004: Posibles escenarios. In: INSTITUTO DE CIENCIA POLÍTICA. Elecciones 1999/2000. Montevideo: Ediciones de la Banda Oriental, 2000. p. 299-322.

JONES, Mark P. Democracy in Latin America, Challenges and Solutions: Political Party and Party System Institutionalization and Women's Legislative Representation. In: Consulta de San José 2007, San José, 16 set. 2007.

JONES, Mark P. Electoral Laws and the Effective Number of Candidates in Presidential Elections. Journal of Politics, v. 61, n. 1, p. 171-184, 1999.

JONES, Mark P. The role of parties and party systems in the policymaking process. In: Seminario State Reform, Public Policies and Policymaking Processes, Washington D.C, 28 feb.-2 mar. 2005. 
KEMAHLIOGLU, Ozge; WEITZ-SHAPIRO, Rebecca; HIRANO, Shigeo. Why Primaries in Latin American presidential Elections? Journal of Politics, v. 71, n. 1, p. 339-352, 2009.

KITSCHELT, Herbert et al. Latin American Party System. New York, NY: Cambridge University Press, 2010.

LAAKSO, Markku; TAAGEPERA, Rein. Effective Number of Parties: A Measure with Application to West Europe. Comparative Political Estudies, v. 12, p. 3-27, 1979.

LANZARO, Jorge (Ed.). La izquierda uruguaya: entre la oposición y el gobierno. Montevideo: Fin de Siglo, 2004.

LUPU, Noam. Voter Partisanship in Latin America. Madison, Wisconsin: Mimeo, 2014. Disponível em: <http://www.noamlupu.com/partisanshipLAV.pdf>. Acesso em: 29 mar. 2014.

MAINWARING, Scott.Presidencialism and Multipartism: The Difficult Combination. Comparative Political Studies, v. 26, n. 2, p. 198-228, 1993.

MAINWARING, Scott; SCULlY, Timothy (Eds.). Building Democratic Institutions. Party Systems in Latin America. Stanford: Stanford University Press, 1995.

MAINWARING, Scott; SHUGART, Matthew S. Presidentialism and Party System. In: Mainwaring, Scott; SHUGART, Matthew S. (Eds.). Presidentialism and Democracy in Latin America. Cambridge: Cambridge University Press, 1997. p. 12-54.

MAINWARING, Scott; TORCAL, Mariano. La institucionalización de los sistemas de partidos y la teoría del sistema partidista después de la tercera ola democratizadora. América Latina Hoy, v. 41, p. 141-173, 2005.

PAYNE, Mark J.; ZOVATTO, Daniel; DÍAZ, Mercedes M. La politica importa: democracia y desarrollo en América Latina. Washington, DC: Banco Interamericano de Desarrollo y Instituto Internacional para la Democracia y la Asistencia Electoral, 2006.

PEDERSEN, Mogens N. The Dynamics of European Party Systems: Changing Patterns of Electoral Volatility. European Journal of Political Research, v. 7, n. 1, p. 1-26, 1979.

PÉREZ PÉREZ, Alberto. La Ley de Lemas. Montevideo: Fundación de Cultura Universitaria, 1970.

SHUGART, Mathew; CAREY, John. Presidents and assemblies: Constitutional Design and Elecotral Dynamics. Cambridge: Cambridge University Press, 1992.

Texto recebido em 23 de janeiro 2014. Aprovado em 25 de março de 2014. 